\title{
Application of quartz crystal nanobalance in conjunction with a net analyte signal based method for simultaneous determination of leucine, isoleucine and valine
}

\author{
Maryam Shojaei ${ }^{1}$, Abdolreza Mirmohseni ${ }^{2}$, Maryam Farbodi ${ }^{2}$ \\ ${ }^{1}$ Department of Natural sciences, Faculty of Animal sciences, University of Tabriz, Tabriz, Iran; ${ }^{2}$ Polymer Research Technology Labo- \\ ratory, Department of Applied Chemistry, Faculty of Chemistry, University of Tabriz, Tabriz, Iran. \\ Email: mshojaei@tabrizu.ac.ir; mirmohseni@tabrizu.ac.ir; farbodi@tabrizu.ac.ir
}

Received 22 June 2009; revised 16 July 2009; accepted 19 July 2009.

\begin{abstract}
The aim of the present investigation was to develop a biosensor for the detection of amino acids, Leucine, Isoleucine and Valine based on a quartz crystal nanobalance. leucine (Leu), isoleucine (Ile), and valine (Val) were selectively determined by quartz crystal nanobalance (QCN) sensor in conjunction with net analyte signal (NAS)-based method called HLA/GO. An orthogonal design was applied for the formation of calibration and prediction sets including Leu, Ile and Val compounds. The selection of the optimal time range involved the calculation of the net analyte signal regression plot in any considered time window for each test sample. The searching of a region with maximum linearity of NAS regression plot (minimum error indicator) and minimum of PRESS value was carried out by applying a moving window strategy. On the base of obtained results, the differences on the adsorption profiles in the time range between 1 and $300 \mathrm{~s}$ were used to determine mixtures of compounds by HLA/GO method. The results showed that the method was successfully applied for the determination of Leu, Ile and Val.
\end{abstract}

Keywords: Quartz Crystal Nanobalance; Net Analyte Signal; Leucine; Isoleucine; Valine; HLA/GO Method

\section{INTRODUCTION}

Maple Syrup Urine Disease (MSUD) is a rare autosomal recessive metabolic disorder affecting the metabolism of amino acids, which occurs due to a deficiency of the activity of the mitochondrial enzyme complex branched-chain 1-2-ketoacid dehydrogenase (BCKD). The main symptom of MSUD is accumulation of the branched chain amino acids (BCAA) leucine (Leu), isoleucine (Ile) and valine (Val) in blood, urine and cerebrospinal fluid. This deficiency results in mental retardation if not detected soon after birth and l-leucine and/or its keto acid are considered to be the main neurotoxic metabolites in MSUD. So, identification and detection of the abnormal levels of these metabolites in urine samples are necessary to diagnosis and therapy of these pathologies [1].

MSUD screening methods based on gas chromatography-mass spectrometry (GC-MS) and tandem mass spectrometry (MS-MS) have been developed. MS-MS has been reported to be a powerful diagnostic tool in MSUD patients. GC-MS and MS-MS have high resolutions that enable them to be used to measure several amino acids simultaneously. However, these instruments are prohibitively expensive, and hospitals in developing countries cannot afford it [2].

In general, the high-performance liquid chromatography (HPLC) method has been devised to measure amino acid levels, but developed methods have disadvantages, which include complex sample preparation and long analysis time [3].

Mass screening emergency for MSUD in childhood demands to develop simple and inexpensive methods with a rapid and quantitative response. Quartz crystal nanobalance $(\mathrm{QCN})$ is a sensing system based on the sorption of analyte on an adsorbent material [4]. The QCN comprises a thin vibrating AT-cut quartz wafer sandwiched between two metal excitation electrodes. When small amounts of mass are adsorbed at the quartz electrode surface, the frequency of the quartz is changed according to the well-known Sauerbrey equation [5]:

$$
\Delta F=-2.26 \times 10^{-6} F_{0}^{2}(\Delta m / A)
$$

where $\Delta F$ is the measured frequency shift, $F_{0}$ the original oscillation frequency of the dry crystal, $\Delta m$ the mass change, $A$ the piezoelectrically active area of the excitation electrodes.

Due to some advantages including low cost, portabil- 
ity and easy on-line analysis, the quartz crystal nanobalance (QCN) sensor is extensively used for the measurements of mass changes in a variety of chemical and biological studies, such as determination of volatile organic compounds [6,7], poisonous compounds [8] and immunoassay $[9,10]$.

In some cases, the major drawback of the sensors based on QCN is a lack of selectivity since along with the analyte, other compounds usually interfere. In other words, there is no discrimination between the sources of the mass changes. To overcome this shortcoming, one approach is the pattern recognition technique that can be used for the data processing of the QCN signals for the simultaneous determination of mixtures of compounds. Multilinear regression (MLR), partial least squares (PLS) and net analyte signal (NAS) are examples of multivariate analytical techniques $[11,12,13]$.

HLA/GO algorithm (Hybrid Linear Analysis presented by Goicoechea and Olivieri), one of the NASbased methods, has been successfully used for resolving multicomponent mixtures. Goicoechea and Olivieri [14] have determined tetracycline in blood serum by using synchronous spectrofluorimetry through the HLA/GO algorithm. This algorithm has also been applied for the simultaneous determination of leucovorin and methotrexate, by spectrophotometric [15] and Sorbic (SOR) and benzoic (BEN) acids in fruit juice samples by using spectroscopic signals [16]. HLA/GO has also been applied to the determination of binary mixtures of amoxycillin and clavulanic acid by stopped-flow kinetic analysis [17].

To our knowledge, no study reported for the detection and determination of Leu, Ile and Val using QCN technique. In the present study, we report the simultaneous determination of Leu, Ile and Val in the solution containing some common urine analytes using polystyrene (PS) coated QCN. NAS is utilized to process the frequency data of the crystal at various times, based on different adsorption dynamics of Leu, Ile and Val on the PS coated QCN.

\subsection{Theory Notation}

An $I \times J$ data matrix $R$ composed of the calibration responses of $I$ samples at $J$ times, a $J \times 1$ vector $s k$ containing the pure adsorption profile of analyte $k$ at unit concentration, and an $I \times 1$ vector $c k$ of calibration concentrations of analyte $k$ are the used matrices and vectors throughout the present work. The net analyte signal (NAS) for analyte $k\left(r^{*} k\right)$ is given by the following equation:

$$
r^{*} k=\left[I-R_{-k}\left(R_{-k}\right)^{+}\right] r=P_{\mathrm{NAS}, k r}
$$

where $r$ is the adsorption profile of a given sample (when $r$ is the profile $s k$ of pure $k$ at unit concentration, Eq.(2) becomes $\left.s^{*} k=P_{\mathrm{NAS}, k} s_{k}\right), I$ is a $J \times J$ unitary matrix,
$R_{-k}$ is a $J \times A$ column space spanned by the adsorption profile of all other analytes except $k\left(R_{-k}{ }^{+}\right.$is the pseudoinverse of $R_{-k}$ and $A$ is the number of factors used to build the model, and $P_{\mathrm{NAS}, k}$ is a $J \times J$ projection matrix which projects a given vector onto the NAS space.

The concentration of component $k$ in an unknown sample is obtained from its adsorption profile $(r)$ as

$$
c_{k}=\frac{s_{k}^{T} \operatorname{Pr}}{s_{k}^{T} P s_{k}}=\frac{s_{k}^{T} P \operatorname{Pr}}{s_{k}^{T} P P s_{k}}=\frac{\left(s_{k}^{*}\right)^{T} r_{k}{ }^{*}}{\left\|s_{k}^{*}\right\|^{2}}
$$

The applied method in this research involves using the mean (uncentred) calibration profile. It is first obtained as

$$
\bar{r}_{c a l}=\frac{1}{I} \sum_{i=1}^{I} r_{i, c a l}
$$

where $r_{i, \text { cal }}$ is the profile for the $i$ th calibration sample. Then the contribution of analyte $k$ is subtracted from the data matrix $R$ in the following way:

$$
R_{-k}=R-\frac{c_{k} \bar{r}_{c a l}^{T}}{\bar{c}_{k, c a l}}
$$

where $\bar{c}_{k, c a l}$ is the mean (uncentred) calibration concentration of analyte $k$. The calculation of net sensitivity $\left(s_{k}{ }_{k}\right)$ is then carried out with the following equation:

$$
s_{k}^{*}=P_{N A S, k}\left[\frac{\bar{r}_{c a l}^{T}}{\bar{c}_{k, c a l}}\right]
$$

\subsection{Selection of Time Window}

In the present work, the selection of the optimum range of time window was made by calculating an error indicator (EI) as a function of a moving window for each prediction sample, using information of the NASRP (called "net analyte signal regression plot"). NASRP is a plot of the elements of the sample vector $\mathbf{r}_{k}{ }_{k}$ versus those of $\mathbf{s}_{k}{ }_{k}$ and should fit a straight line through the origin, with random residuals and slope $c k$. Large and correlated residuals in this plot reveal discrepancies between the measured profile (and thus in $\mathbf{r}^{*} k$ ) and the model and, possibly, bias in the estimated concentration. The expression for EI used in the present context is [17]:

$$
E I=\frac{\left[s^{2}\left(1+\frac{N^{2} s^{2}}{4\left\|r^{*}\right\|^{2}}\right)\right]^{1 / 2}}{\left\|r^{*}\right\|}
$$

where $s$ is the standard deviation from the best-fitted straight line to the NASRP (in a given adsorption region), 
and $N$ is the number of points in the latter plot.

\section{MATERIALS AND METHODS}

\subsection{Reagents and Material}

All reagents used in this experiment were of analytical grade. leucine (Leu), isoleucine (Ile), valine (Val) were from Sigma chemicals with analytical grade. Polystyrene (PS) was supplied by Tabriz Petrochemical Co., Iran.

\subsection{Instrumentation}

$10 \mathrm{MHz}$ AT-Cut quartz crystals with gold coating on both sides were commercially available from International Crystal Manufacturer (ICM, Oklahoma, USA). For QCN experiments a home made apparatus was used as described in our previous work [18].

\subsection{Procedures}

A solution casting method was used to coat the polymer over the quartz crystal electrode. Using a Hamilton micro liter syringe (Hamilton BonaduzAG, Switzerland), $4 \mu \mathrm{L}$ of PS/chloroform solution $(0.3 \%, \mathrm{w} / \mathrm{v})$ was dropped on top of the gold electrode of the quartz crystal. A thin layer of PS was obtained after solvent evaporation.

An orthogonal design was applied for the formation of calibration and prediction sets including Leu, Ile and Val. Orthogonal design is used in order to give the most information from the analytical system by using only a few samples. The calibration and prediction sets were prepared according to four-level orthogonal design.

The concentrations varied in the linear range of each compound (50-300 mgL ${ }^{-1}$ for Leu, $100-400 \mathrm{mgL}^{-1}$ for Ile and Val). All solutions were filtered using a syringe filter $(0.2 \mu \mathrm{m})$ before injecting to the cell. Milli-Q water was used to desorbed analyte and recover the electrode. All measurements were carried out at room temperature $\left(25^{\circ} \mathrm{C}\right)$.

\section{RESULTS AND DISCUSSION}

\subsection{Determination of Pure Leu}

The polymer-coated electrode was exposed to a constant concentration of aqueous Leu solution $\left(50 \mathrm{mgL}^{-1}\right)$. Typical responses for Leu are shown in Figure 1. The frequency of the crystal decreased due to the adsorption of analyte to the surface of polymer modified electrode according to Eq.(1). The recorded responses showed that the electrode is sensitive to Leu. The frequency of the crystal was back shifted to its initial value by exposure to the Milli-Q water indicating the full description of analyte from the electrode surface (Figure 1).

As the concentration of analyte increased the magnitude of the response increased (Figure 2), the calibration

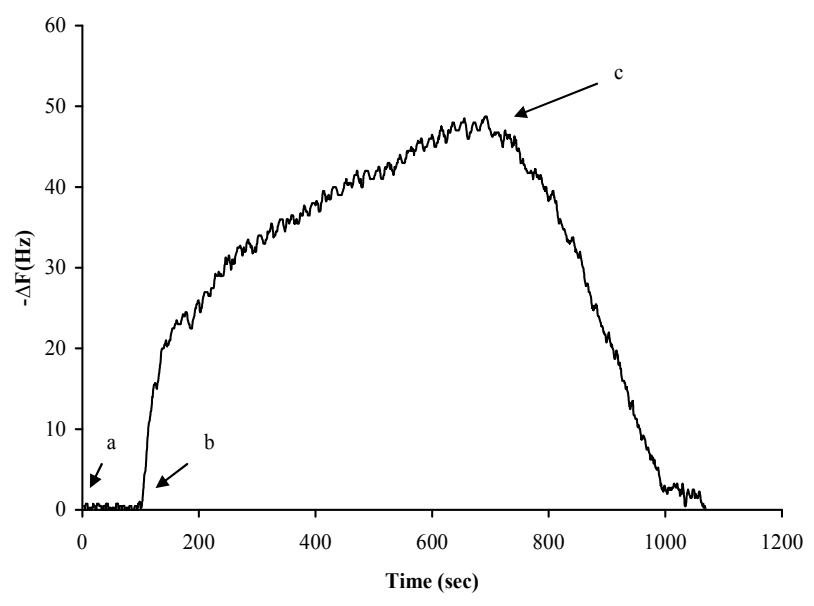

Figure 1. Typical frequency change of PS modified quartz crystal electrode recorded upon exposure to a Leu solution $\left(100 \mathrm{mgL}^{-1}\right)$. (a) Milli-Q water, (b) Leu solution $\left(100 \mathrm{mgL}^{-1}\right)$, (c) Milli-Q.

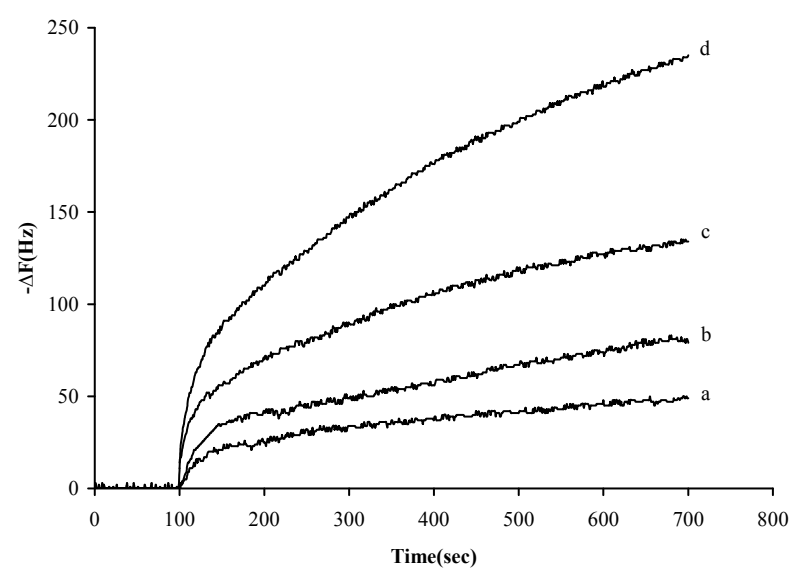

Figure 2. Frequency changes of PS modified quartz crystal electrode as a function of time exposed to various concentration of Leu solutions: (a) $50 \mathrm{mgL}^{-1}$, (b) $100 \mathrm{mgL}^{-1}$, (c) 200 $\mathrm{mgL}^{-1}$, (d) $300 \mathrm{mgL}^{-1}$.

curve was constructed by plotting the frequency shifts against the concentration of Leu (Figure 3). The responses were linear against Leu concentrations in the range $50-300 \mathrm{mgL}^{-1}$ and with linear regression coefficient of $0.9806(n=4)$.

\subsection{Determination of Leu in the Presence of Ile and Val}

Based on the above results, the QCN sensor coated with PS can be employed as pure Leu sensor. Since Leu, Ile and Val are considered as the agents that exist simultaneously in the most urine samples of MSUD patients, it is necessary to investigate the cross-sensitivity between Leu, Ile and Val. So, the frequency shifts were recorded for quartz crystal PS-coated electrode upon exposure Ile and Val. The linear range was obtained $100-400 \mathrm{mgL}^{-1}$ for Ile and Val. The frequency shift obtained for quartz 


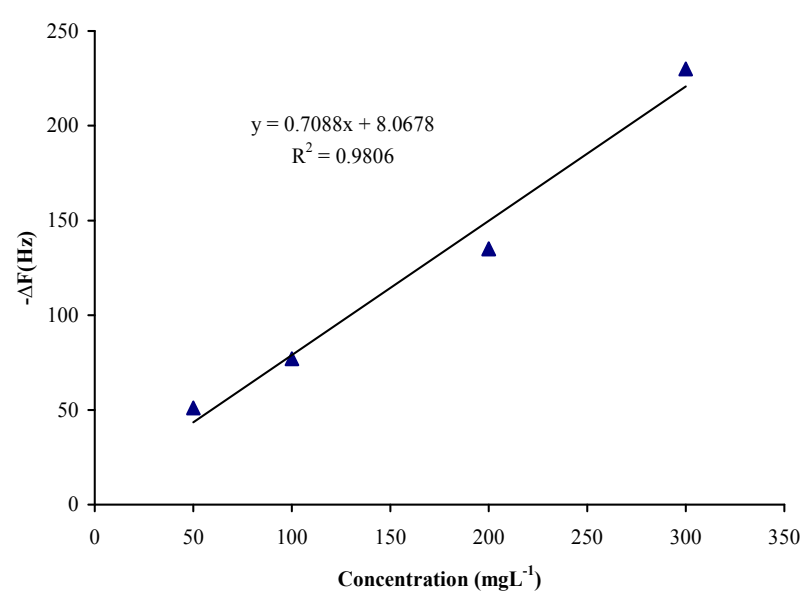

Figure 3. Calibration graph for Leu solutions exposed to PS modified quartz crystal electrode. Exposure time: 10 min.

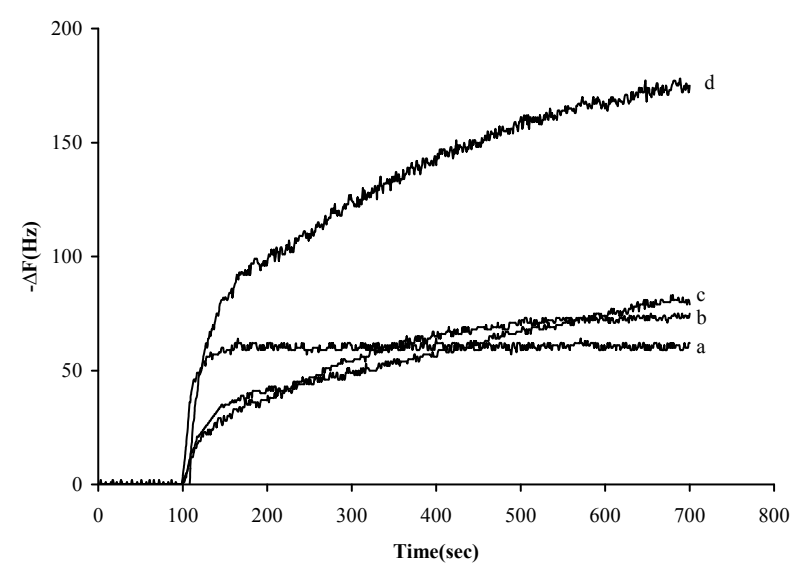

Figure 4. PS modified QCN sensor response upon exposure to: (a) Leu at the concentration of $100 \mathrm{mgL}^{-1}$, (b) Ile at the concentration of $160 \mathrm{mgL}^{-1}$, (c) Val at the concentration of 130 $\mathrm{mgL}^{-1}$, (c) mixture of Leu $\left(100 \mathrm{mgL}^{-1}\right) / \mathrm{Ile}\left(160 \mathrm{mgL}^{-1}\right)$ /Val $\left(130 \mathrm{mgL}^{-1}\right)$.

crystal PS-coated electrode upon exposure to Leu (100 $\left.\mathrm{mgL}^{-1}\right)$, Ile $\left(160 \mathrm{mgL}^{-1}\right)$, Val $\left(130 \mathrm{mgL}^{-1}\right)$ and a mixture of Leu $\left(100 \mathrm{mgL}^{-1}\right) / \mathrm{Ile}\left(160 \mathrm{mgL}^{-1}\right) /$ Val $\left(130 \mathrm{mgL}^{-1}\right)$ were recorded (Figure 4$)$. The concentrations were selected from the linear range of each compound. The obtained responses showed a significant change in the shape of the frequency-time curves of Leu with including Ile and Val (Figure 4). Then, net analyte signal-based HLA/GO method was considered to develop a model for selectively determination of Leu, Ile and Val compounds.

\subsection{Optimization of HLA/GO Method}

Selection of the optimum number of factors to be used within the HLA/GO algorithms allows one to model the system with the optimum amount of information. In HLA/GO analysis of the calibration set, the PRESS value (predicted error sum of squares) for prediction samples varies as a function of the number of factors. In the present work, cross-validation has been used to select the optimum number of factors for two time intervals, in the range comprised between 1 and $600 \mathrm{~s}$.

The selection of the optimum time region is caused to increase the predictive ability of multivariate analysis by discarding the non-informative parts of adsorption profile from the original data.

The selection of the optimum time region for the analysis was carried out by evaluating the best predicted values for the prediction samples and the minimum error EI values. In this regard, using the optimized number of factors selected in each region, an EI was calculated for each prediction sample, using information of the NASRP.

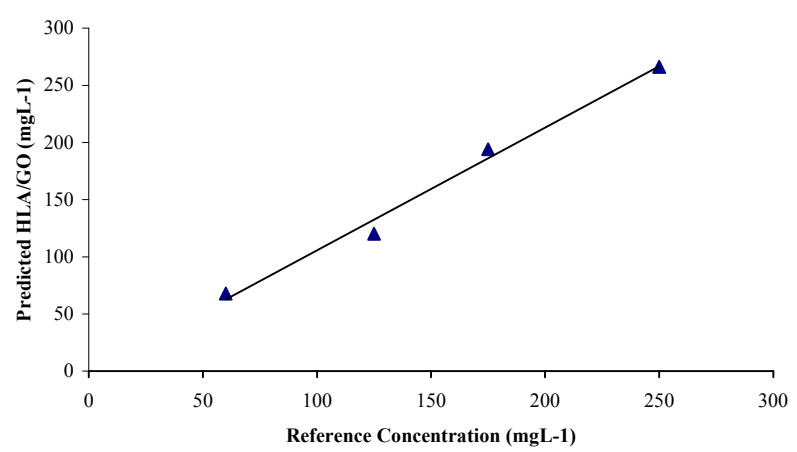

(a)

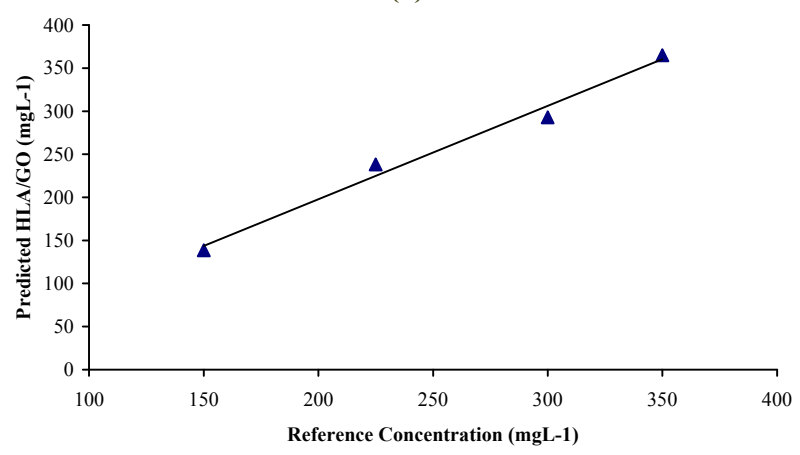

(b)

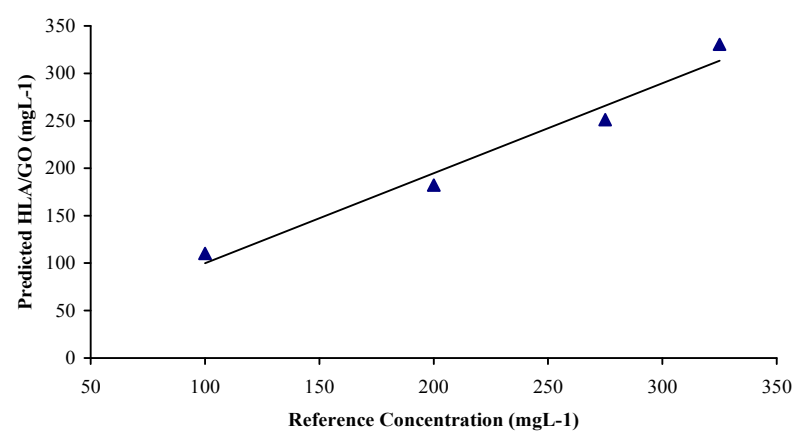

(c)

Figure 5. Predicted vs. actual concentrations for HLA/GO calibration models (a) Leu, (b) Ile and (c) Val. 
Table 1. Optimization of the sensor range in the prediction of Leu, Ile and Val in the mixture by application of the NAS signal and evaluation of the EI. ( ${ }^{1}$ actual concentration; ${ }^{2}$ predicted concentration)

\begin{tabular}{|c|c|c|c|c|c|c|c|c|c|c|c|c|c|c|}
\hline Sample & $\begin{array}{c}\text { Time } \\
\text { Range }\end{array}$ & Factor & EI & Leu $^{1}$ & Leu $^{2}$ & Error & EI & Ile $^{1}$ & $\mathrm{Ile}^{2}$ & Error & EI & Val $^{1}$ & $\mathrm{Val}^{2}$ & Error \\
\hline \multirow[t]{2}{*}{1} & $1-300$ & 3 & 0.43 & 60 & 67.7 & 7.7 & 0.04 & 325 & 330.6 & 5.61 & 0.57 & 300 & 293.1 & -6.8 \\
\hline & $1-600$ & 5 & 0.56 & & 43.8 & -16.5 & 0.04 & & 346.1 & 21.1 & 0.57 & & 317.1 & 17.1 \\
\hline \multirow[t]{2}{*}{2} & $1-300$ & 3 & 0.42 & 125 & 120.1 & -5.1 & 0.08 & 275 & 251.1 & -13.86 & 0.06 & 350 & 365.0 & 15.06 \\
\hline & $1-600$ & 5 & 0.36 & & 146.0 & 21.0 & 0.05 & & 142.6 & -22.23 & 0.77 & & 370.9 & 20.9 \\
\hline
\end{tabular}

The moving window was obtained by varying the time range. Table 1 shows the ranges of time tested, the optimum number of factors for each region, the EI values calculated, and the predicted values for Leu, Ile and Val. The minimum EI value calculated using information of the NASRP indicates $1-300 \mathrm{~s}$ as the most adequate time region for the analysis in this case.

The optimized model was tested in the analysis of the prediction set and plots of $c_{\text {pred }}$ versus $c_{\text {act }}$ were constructed (Figure 5). As it can be seen, the plot showed very good linearity and the values of $0.9894,0.9707$ and 0.9854 were obtained as correlation coefficient for Leu, Ile and Val, respectively.

\section{CONCLUSIONS}

Leucine (Leu), isoleucine (Ile), valine (Val) were simultaneously determined using adsorption profile data recorded using PS-coated QCN sensor in conjunction with HLA/GO multivariate calibration method. Determination was based on frequency shifts of PS modified quartz crystal electrode due to the adsorption of Leu at the surface of modified electrode in the presence of Ile and Val. The responses were linear against Leu concentrations in the range $50-300 \mathrm{mgL}^{-1}$ and with linear regression coefficient of $0.9806(n=4)$, respectively. The selection of optimum time ranges for each analyte separately were performed by getting the minimum EI, based on the minimization of the PRESS, as a function of a moving adsorption time window. The analysis of the prediction set was used to test the optimized model and plots of $c_{\text {pred }}$ versus $c_{\text {act }}$ showed very good linearity. The values of $0.9894,0.9707$ and 0.9854 were obtained as correlation coefficient for Leu, Ile and Val, respectively.

\section{ACKNOWLEDGMENTS}

We are most grateful the financial supports of this research project by the University of Tabriz.

\section{REFERENCES}

[1] Barschak, A. G., Marchesan, C., Sitta, A., Deon, M., Giugliani, R., Wajner, M., and Vargas, C. R., (2008) Maple syrup urine disease in treated patients: Biochemical and oxidative stress profiles, Clinical Biochemistry, 41, 317-324.

[2] Deng, C. and Deng, Y., (2003) Diagnosis of maple syrup urine disease by determination of 1-valine, 1-isoleucine, 1-leucine and 1-phenylalanine in neonatal blood spots by gas chromatography-mass spectrometry, Journal of Chromatography B, 792, 261-268.

[3] Bridi, R., Fontella, F. U., Pulrolnik, V., Braun, C. A., Zorzi, G. K., Coelho, D., Wajner, M., Vargas, C. R., and Dutra-Filho, C. S., (2006) A chemically-induced acute model of maple syrup urine disease in rats for neurochemical studies, Journal of Neuroscience Methods, 155, 224-230.

[4] Lau, K. T., Micklefield, J., and Slater, J. M., (1998) The optimization of sorption sensor array for use in ambient conditions, Sens. Actuators. B, Chem., 50, 69-79.

[5] Sauerbrey, G. Z., (1959) The use of quartz oscillators for weighing thin layers and for microweighing, Phys, 155, 206-222.

[6] Mirmohseni, A. and Oladegaragoze, A., (2004) Determination of chlorinated aliphatic hydrocarbons in air using a polymer coated quartz crystal microbalance sensor Sensors and Actuators B, 102, 261-270.

[7] Mirmohseni, A. and Oladegaragoze, A., (2003) Determination of Ammonia and Aliphatic amines in Air Using Poly(N-vinylpyrrolidone) Coated Quartz Crystal Microbalance Sensors and Actuators B, 89, 164-172.

[8] Mirmohseni, A. and Alipour, A., (2002) Construction of a sensor for the determination of cyanide in industrial effluents: A method based on Quartz Crystal Microbalance Sensors and Actuators B, 84, 245-251.

[9] Mirmohseni, A., Shojaei, M., and Farbodi, M., (2008) Application of a quartz crystal nanobalance to the molecularly imprinted recognition of phenylalanine in solution Biotechnology and bioprocess Engineering, 13, 592-597.

[10] Shojaei, M., Mirmohseni, A., and Farbodi, M., (2008) Application of a quartz crystal nanobalance and principal component analysis for the detection and determination of histidine, Anal. Bioanal. Chem., 391, 2875-2880.

[11] Zhu,W., Wei,W., Nie, L., and Yao, S., (1993) Anal. Chim. Acta, 282, 535-541.

[12] Nyberg, H., (2008) Multivariate analysis applied to a test procedure for determining gun propelling charge weight Part II, Partial least squares analysis, Chemometrics and Intelligent Laboratory Systems, 92, 118-124.

[13] Mirmohseni, A., Abdollahi, H., and Rostamizadeh, K., (2007) Net analyte signal-based simultaneous determination of ethanol and water by quartz crystal nanobalance sensor, Analytica Chimica Acta, 585, 179-184.

[14] Goicoechea, H. C. and Olivieri, A. C., (1999) Enhanced synchronous spectrofluorometric determination of tetracycline in blood serum by chemometric analysis. Comparison of partial least-squares and hybrid linear analysis calibrations, Anal. Chem., 71, 4361-4368.

[15] Espinosa-Mansilla, A., Meras, I. D., Gomez, M. J. R., 
Munoz de la Pena, A., and Salinas, F., (2002) Selection of the wavelength range and spectrophotometric determination of leucovorin and methotrexate in human serum by a net analyte signal based method Talanta, $\mathbf{5 8}$, 255-263.

[16] Marsili, N. R., Sobrero, M. S., and Goicoechea, H. C., (2003) Spectrophotometric determination of sorbic and benzoic acids in fruit juices by a net analyte signal-based method with selection of the wavelength range to avoid non-modelled interferences, Anal. Bioanal. Chem., 376, 126-133.
[17] Munoz de la Pena, A., Espinosa-Mansilla, A., Acedo Valenzuela, M. I., Goicoechea, H. C., and Olivieri, A. C., (2002) Comparative study of net analyte signal-based methods and partial least squares for the simultaneous determination of amoxycillin and clavulanic acid by stopped-flow kinetic analysis, Anal. Chim. Acta, 463, 75-88.

[18] Mirmohseni, A., Milani, M., and Hassanzadeh, V., (1999) Ion exchange properties of polypyrrole studied by electrochemical quartz crystal microbalance (EQCM), Polym. Int., 48, 873-878. 